Journal of

Synchrotron

Radiation

ISSN 0909-0495

Received 15 June 2010

Accepted 15 October 2010

\section{A new paradigm for macromolecular crystallo- graphy beamlines derived from high-pressure methodology and results}

\author{
Roger Fourme, ${ }^{a *} \ddagger$ Eric Girard, ${ }^{b} \ddagger$ Anne-Claire Dhaussy, ${ }^{c}$ Kadda Medjoubi, $^{a}$ \\ Thierry Prangé, ${ }^{d}$ Isabella Ascone, ${ }^{e}$ Mohamed Mezouar ${ }^{f}$ and Richard Kahn ${ }^{b}$ \\ ${ }^{\mathbf{a}}$ Synchrotron SOLEIL, BP 48, Saint Aubin, 91192 Gif-sur-Yvette, France, ${ }^{\mathbf{b}}$ IBS (UMR 5075

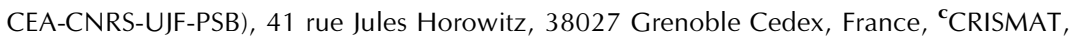 \\ ENSICAEN, 6 Boulevard du Maréchal Juin, 14000 Caen, France, 'LCRB (UMR 8015 CNRS), \\ Université Paris Descartes, Faculté de Pharmacie, 4 avenue de l'Observatoire, 75270 Paris, \\ France, ${ }^{\mathbf{e} E N S C P}$ (UMR CNRS 7223), 11 rue Pierre et Marie Curie, 75231 Paris Cedex 05, \\ France, and ${ }^{\mathrm{f} E S R F}$, BP 220, 38043 Grenoble, France. E-mail: roger.fourme@synchrotron-soleil.fr
}

\begin{abstract}
Biological structures can now be investigated at high resolution by high-pressure X-ray macromolecular crystallography (HPMX). The number of HPMX studies is growing, with applications to polynucleotides, monomeric and multimeric proteins, complex assemblies and even a virus capsid. Investigations of the effects of pressure perturbation have encompassed elastic compression of the native state, study of proteins from extremophiles and trapping of higher-energy conformers that are often of biological interest; measurements of the compressibility of crystals and macromolecules were also performed. HPMX results were an incentive to investigate short and ultra-short wavelengths for standard biocrystallography. On cryocooled lysozyme crystals it was found that the data collection efficiency using $33 \mathrm{keV}$ photons is increased with respect to $18 \mathrm{keV}$ photons. This conclusion was extended from $33 \mathrm{keV}$ down to $6.5 \mathrm{keV}$ by exploiting previously published data. To be fully exploited, the potential of higher-energy photons requires detectors with a good efficiency. Accordingly, a new paradigm for MX beamlines was suggested, using conventional short and ultra-short wavelengths, aiming at the collection of very high accuracy data on crystals under standard conditions or under high pressure. The main elements of such beamlines are outlined.
\end{abstract}

Keywords: macromolecular crystallography; high pressure; short X-ray wavelength.

\section{Introduction}

High-pressure (HP) molecular biophysics is a developing field. Our planet is generating a piezophilic environment since most of the biosphere is characterized by pressure greater than $10 \mathrm{MPa}(100 \mathrm{MPa}=0.1 \mathrm{GPa}=1 \mathrm{kbar})$. The recent discovery of the first obligate piezophile archae, Pyrococcus yayanosii (Zeng et al., 2009), illustrates the interest of high-pressure life adaptation. Concomitant to the understanding of life adaptation to high pressure, the question of the origin of life is raised. The potential throughput for biotechnological developments as well as for environmental questions is of societal interest. Pressure perturbation is a mild and efficient tool for exploring the whole spectrum of conformers of proteins and other biomolecules, from the native folded state to unfolded states. On the basis of differences in partial specific volume,

$\ddagger$ These authors contributed equally to this work. higher-energy conformers of biological relevance can be selectively promoted by high pressure (Akasaka, 2006).

A better understanding of the fundamental mechanisms responsible for the effects of $\mathrm{HP}$ on biomolecules requires high-resolution structural information, which is still scarce. For a few years, such information has become accessible with the implementation of pressure perturbation in both NMR and macromolecular crystallography (MX). Up to 2002, only few crystal structures of small proteins at high pressure were published, using a beryllium cell (Kundrot \& Richards, 1987; Urayama et al., 2002). The lack of structural data at high pressure was due mainly to the cumulated complexities of high-pressure containment and crystallography and to the lack of interest in such studies. A technical breakthrough was achieved with a set-up at the ESRF ID30 then ID27 beamlines, combining a diamond-anvil cell (DAC), ultra-short-wavelength X-rays from undulators and a large area detector (Fourme et al., 2001). The accessible pressure range was 
increased by almost one order of magnitude with respect to beryllium cells, exceeding $2 \mathrm{GPa}$. The quality of diffraction data collected under high pressure achieved usual standards (Girard et al., 2007a).

The main technical advances of high-pressure macromolecular crystallography (HPMX) will be recalled and a summary description of recent scientific results will illustrate its contribution to structural studies and for determining crystal and molecular compressibility, demonstrating that it can now be considered as a mature and general technique (Fourme et al., 2009). HPMX instrumentation and methods have contributed to a renewed interest for short and ultrashort wavelengths, ${ }^{1}$ not only for HP studies but also for standard data collection. It is shown that, in addition to well documented advantages of these unusual wavelengths, the data collection efficiency (DCE), defined as the amount of diffraction data of a given resolution that can be acquired per crystal unit-volume (Fourme et al., 2003), increases at shorter wavelengths. These results consolidate the scientific case for building synchrotron radiation beamlines dedicated to shortwavelength data collection and HPMX.

\section{HPMX instrumentation}

Two crucial components of a HPMX set-up are (i) the highpressure cell and (ii) an intense, parallel (or slightly convergent) and highly monochromatic X-ray beam of short or, better, ultra-short wavelength. Recent high-pressure cells for HPMX are purposely designed DACs. They feature a large useful opening angle $\left(\sim 85^{\circ}\right)$ for both incoming and diffracted beams and the thrust applied to diamonds is generated by a pneumatic built-in system, either a membrane (Girard et al., 2007a) or a piston (Girard et al., 2010a) connected to an external source of pressurized gas. A single crystal with dimensions up to $350 \times 350 \times 150 \mu \mathrm{m}$, or alternatively several smaller crystals, can be compressed from atmospheric pressure to >2 GPa; temperature is adjustable from 293 to $393 \mathrm{~K}$ (Girard et al., 2010a). Currently, routine HPMX data collection with synchrotron radiation is available only at the $6 \mathrm{GeV}$ ESRF storage ring, on the ID27 beamline and more recently FIP-BM30A. ID27 is a high-pressure diffraction beamline with a pair of in-vacuum undulators. As this multipurpose beamline is overbooked, only a small fraction of available beam time is allocated to HPMX, which limits the number of accepted proposals and practically excludes methodological programs requiring systematic tests. FIP-BM30A is a French CRG MX beamline on a bending magnet. Albeit not optimal for HPMX in terms of maximum useful photon energy, brightness and divergence, an interesting feature of this beamline is a six-axis robotic arm normally used as an automated sample changer. Mechanics and software were modified in order to handle the DAC, including automatic centring of the compressed crystal. The robotic arm acts as a goniometer

\footnotetext{
${ }^{1}$ Nomenclature: ultra-long wavelengths, $\lambda \geq 3 \AA$; long wavelengths, $2 \AA \leq \lambda<$ $3 \AA$; conventional wavelengths, $1 \AA \leq \lambda<2 \AA$. Short wavelengths, $0.5 \AA \leq \lambda<$ $1 \AA$; ultra-short wavelengths, $\lambda<0.5 \AA$, as proposed by Helliwell et al. (1993).
}

for data collection by the oscillation method. Successful results were recently achieved (Girard et al., 2010a).

The third most important component of a HPMX set-up is the area detector. On FIP-BM30A, data were collected at short wavelength (photon energy $\simeq 18 \mathrm{keV}$ ) using a Quantum 315R CCD detector (ADSC, USA). Experiments on ID27 at ultra-short wavelengths were initially performed using a Mar345 imaging plate detector (Marresearch, Germany). The large area of this detector allows the crystal-to-detector distance to be increased for a given resolution, which, with a parallel X-ray beam, improves the signal-to-noise ratio at a given wavelength (Schiltz et al., 1997). Another useful characteristic of the imaging plate is a sensitive coating that contains barium. By adjusting the photon energy just over the $\mathrm{Ba} K$-edge at $37.414 \mathrm{keV}$, the detector acts as an energy filter because the detective quantum efficiency (DQE) is maximal for elastic scattering and reduced for Compton scattering (Fourme et al., 2001). Good results were obtained with this detector, albeit that the long readout time is a major drawback for HPMX data collection. The imaging plate was replaced by a Mar165 CCD detector (Rayonix, USA), which approximately doubled the data collection rate. The DQE of the Mar165 CCD detector with the standard phosphor ${ }^{2}$ is $\sim 0.55$ and $\sim 0.14$ at 18 and $33 \mathrm{keV}$, respectively (data from Rayonix). All photons shining on the sample contribute to sample degradation, but only a fraction (given by the detector DQE) is useful for diffraction. With a low-DQE detector, crystals are considerably over-irradiated and submitted during longer exposure times to damages from radicals produced by irradiation.

\section{HPMX results}

As a general rule, any good quality macromolecular crystal loaded in the DAC can be compressed without degradation of three-dimensional order, up to a certain (crystal-specific) range of pressure where diffraction is reduced and finally lost. This remarkable behaviour was attributed to the peculiar nature, half-liquid and half-solid, of macromolecular crystals, which ensures substantial plasticity and hydrostatic compression of molecules thanks to solvent channels in the crystal. We have compressed crystals of various polypeptide, proteins and macromolecular assemblies, some of them beyond $1 \mathrm{GPa}$ and sometimes up to $2 \mathrm{GPa}$, without substantial reduction of diffracting power (Fig. 1, Table 1). Observed effects of pressure include (i) elastic compression of the native sub-state (Girard et al., 2005), (ii) higher-energy sub-states of biological interest where modifications of the native state were of small amplitude, mainly at the active site (Girard et al., 2010b; Ascone et al., 2010b), (iii) dissociation of an oligomeric protein (Girard et al., 2010b) and (iv) anisotropic compression of protein backbone and of side chains (Ascone et al., 2010b). HPMX has also been used to measure, in addition to crystal

\footnotetext{
${ }^{2} \mathrm{Gd}_{2} \mathrm{O}_{2} \mathrm{~S}: \mathrm{Tb}$, thickness $40 \mu \mathrm{m}$, and assuming an effective density of $4.5 \mathrm{~g} \mathrm{~cm}^{-3}$
} 
Table 1

Results of HPMX studies where data collection at one or several pressures was completed.

$N=$ number of crystals. Res $=$ resolution. Compl $=$ completeness. Red $=$ redundancy. $\mathrm{PDB}=$ Protein Data Bank. IP $=$ imaging plate.

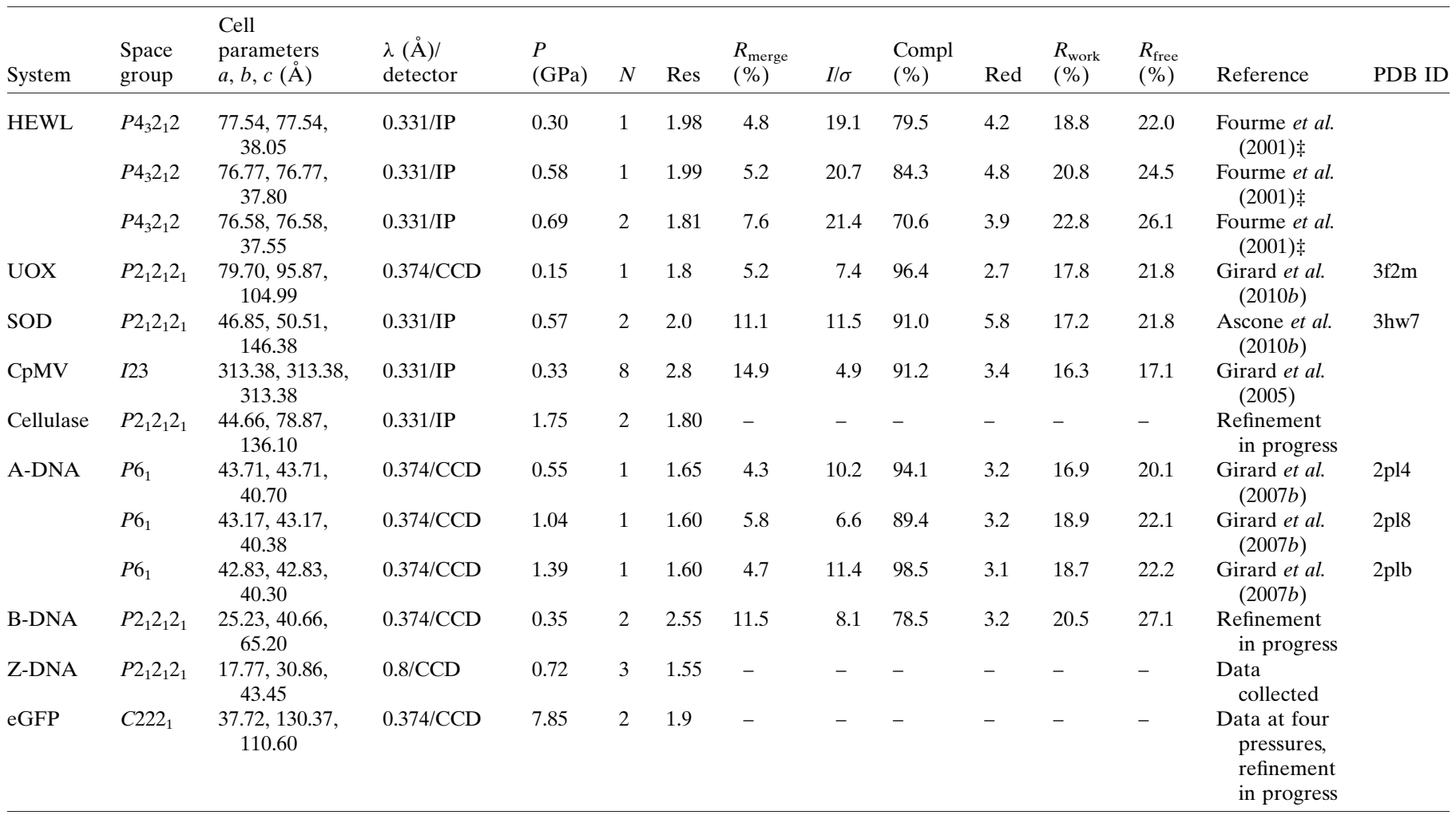

† Structure refinements by T. Prangé (private communication).

compressibility, the intrinsic molecular compressibility of several polypeptides and proteins (Ascone et al., 2010a).

The accuracy and completeness of diffraction data as well as refinement results have reached in most cases standards of conventional MX (Table 1).

\section{Shorter wavelengths increase data collection efficiency}

The interest for macromolecular crystallography in short and ultra-short wavelengths emitted by bright synchrotron sources was strongly advocated by Helliwell et al. (1993), who showed that data sets of unprecedented quality could be obtained at these wavelengths by reducing random and systematic errors in conjunction with very high values for completeness and multiplicity. Gonzalez et al. (1994) did not find improvement in the signal-to-noise ratio at $0.55 \AA$ with respect to $0.92 \AA$. Schiltz et al. (1997) performed the first complete experiment at ultra-short wavelength, exploiting anomalous scattering near the Xe $K$-edge for phasing. Fourme et al. (2003), in the context of the use of ultra-short wavelengths in particular for HPMX, discussed ways to improve the signal-to-noise ratio in a particular diffraction experiment, in order to increase the DCE. Whether the DCE is improved at higher energy for data collected under essentially the same experimental conditions on a given sample remained a matter of controversy.
We have investigated this question from a practical point of view on cryocooled crystals at atmospheric pressure. Hen eggwhite lysozyme (HEWL) crystals (space group $P 4_{3} 2_{1} 2$ ) were grown at $293 \mathrm{~K}$ in a solution containing $\mathrm{NaCl}(1.6 \mathrm{M})$ and sodium acetate buffer $(100 \mu \mathrm{L}) \mathrm{pH} 4.5$. Samples from the same batch and with similar dimensions were mounted in loops and cryocooled in a stream of cold nitrogen at $100 \mathrm{~K}$. Experiments were performed on the ID27 beamline using unfocused monochromatic beam from the $\mathrm{Si}(111)$ channel-cut monochromator adjusted at the zirconium or iodine $K$-edge (17.997 keV and $33.168 \mathrm{keV}$, respectively). The crystal-todetector distance of the Mar165 CCD detector was set to obtain the same resolution at the detector edge for both energies. Data sets were collected over $90^{\circ}$ in $1^{\circ}$ steps. Exposure times were adjusted in order to collect data with the same average signal-to-noise ratio and resolution $[1.5 \AA$ at $I / \sigma(I) \geq 2$ ]. At $18 \mathrm{keV}, 44$ sets were successively collected on the same crystal. While rotating over the same full angular range, the sample was irradiated for a time corresponding to the collection of $\sim 40$ additional data sets, and a full data set was then recorded. The same procedure was repeated once. Data were integrated using the program XDS (Kabsch, 1993) and average $B$-factor values were obtained from Wilson plots calculated by the program. In Fig. 2, $B$ values are plotted as a function of data set number for the complete data collection at $18 \mathrm{keV}$, showing a linear increase over a total irradiation corresponding to $\sim 124$ data sets. At $33 \mathrm{keV}$, owing to longer 


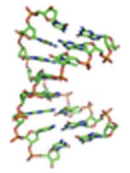

$A-D N A$ $1.9 \mathrm{GPa}$

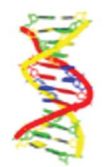

$B-D N A$ $0.5 \mathrm{GPa}$

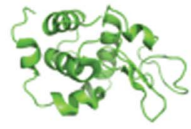

HEWL

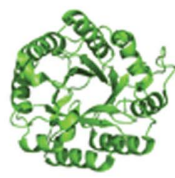

Cellulase 1.8 GPa

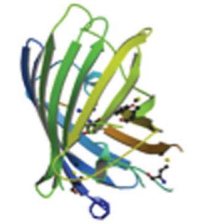

eGFP

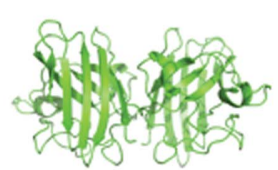

Cu,Zn SOD

Figure 1 $1 \mathrm{GPa}$

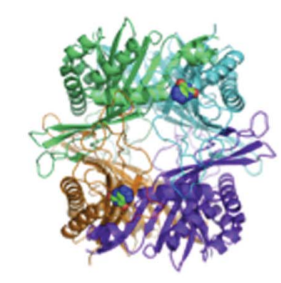

Urate oxidase

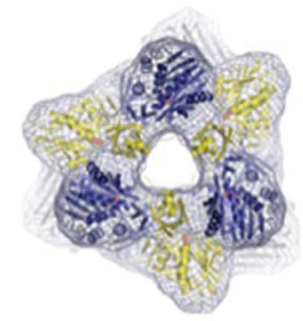

TET protease $0.3 \mathrm{GPa}$

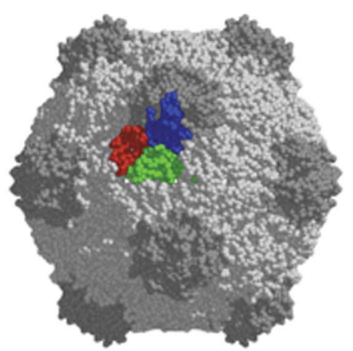

Cowpea Mosaic Virus $0.35 \mathrm{GPa}$

Selected examples of macromolecular structures investigated by HPMX (from works by authors of this article and co-workers).

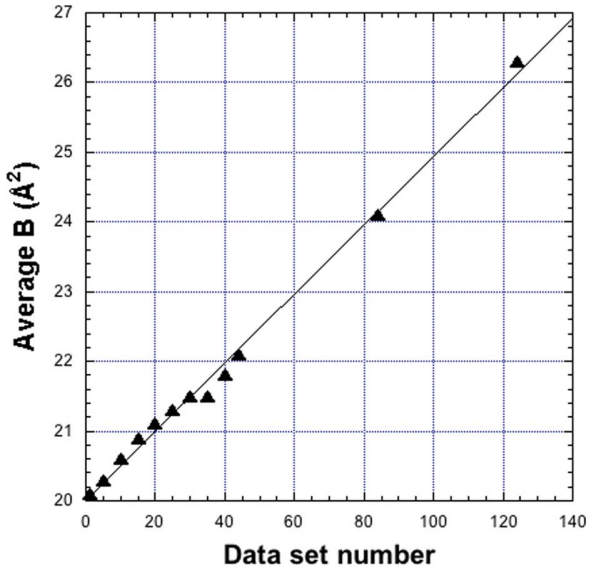

Figure 2

Variation of the average $B$ factor as a function of data set number during multiple data acquisition with $18 \mathrm{keV}$ photons and a CCD detector.

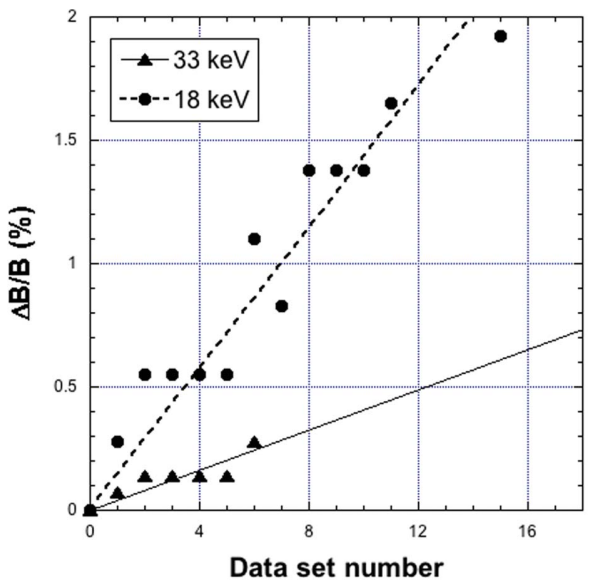

Figure 3

Intrinsic $\Delta B / B$ variation as a function of data set number during repeated acquisitions on a cryocooled HEWL crystal at 18 and $33 \mathrm{keV}$. The first data set is labelled 0 and the $18 \mathrm{keV}$ plot is limited to the first 16 data sets of the complete collection shown in Fig. 2. exposure time and limited available beam time, seven data sets only were collected, showing also a linear increase of $B$ values. Interestingly, slopes at 18 and $33 \mathrm{keV}, 0.226$ and $0.285 \%$ per data set, respectively, are similar in spite of the fact that the $\mathrm{DQE}$ at $33 \mathrm{keV}$ is divided by $\sim 4$ with respect to $18 \mathrm{keV}$ (see $\S 2)$. In Fig. 3, experimental $\Delta B / B$ values were multiplied by the detector DQE at the relevant wavelength, which gives the (intrinsic) variation which would be observed with an ideal detector $(\mathrm{DQE}=1)$. The slopes of fitted linear curves in Fig. 3 are 0.041 and $0.143 \%$ per data set at 33 and $18 \mathrm{keV}$, respectively. Accordingly, assuming an ideal detector, the number of data sets of the prescribed quality that could be collected on a single HEWL crystal would be multiplied by $\sim 3.5$ at $33 \mathrm{keV}$ when compared with $18 \mathrm{keV}$. The actual intrinsic gain at $33 \mathrm{keV}$ is probably even larger, given that longer exposures (necessary to compensate for the low DQE of the detector at $33 \mathrm{keV}$ ) increase the residence time in the sample of aggressive radicals produced by irradiation.

Shimizu et al. (2007) have reported measurements of successive $180^{\circ}$ data sets ( 12 to 15 ) collected on a cryocooled HEWL crystal at nine X-ray energies from 6.5 to $33 \mathrm{keV}$ using a Quantum 315 (ADSC, USA), a multi-CCD detector with the same type of phosphor as the Mar165 CCD. These authors were mainly interested in the dose dependence of radiation damage with photon energy. At each energy, average $B$ factors were derived from a Wilson plot for the first and last data set. We analyzed these data assuming again DQE $=1$. We found that the relative variation of $B$ per data set was decreasing from $6.5 \mathrm{keV}$ to $33 \mathrm{keV}$ (Fig. 4), thus consolidating and extending to a much broader energy range conclusion drawn from our measurements at 18 and $33 \mathrm{keV}$. Systematic and random errors during data collection are smaller when using shorter wavelengths (Helliwell et al., 1993; Fourme et al., 2003). Results presented in this article for HEWL crystals suggest that, in addition, more data can be collected on a given 


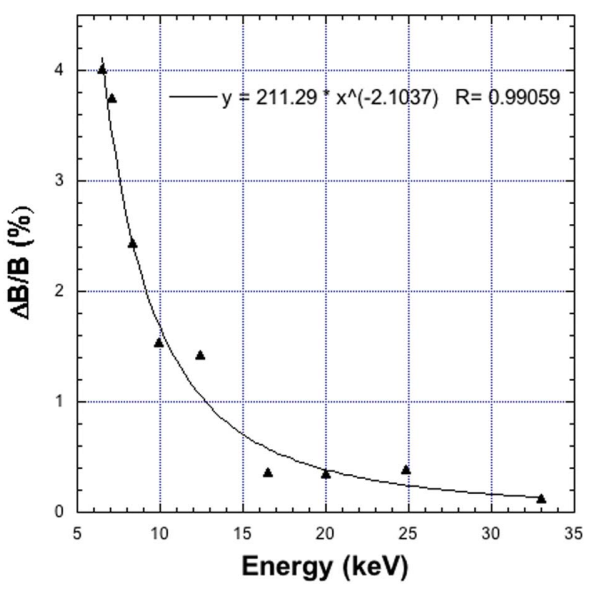

Figure 4

Variation of $\Delta B / B$ per data set versus energy during repeated data acquisitions on a cryocooled HEWL crystal performed at nine photon energies from 6.5 to $33 \mathrm{keV}$ with a CCD detector. Analysis is based on Table 1 of Shimizu et al. (2007), after rescaling assuming DQE $=1$ over the whole energy range.

cryocooled sample, which gives access to, for example, longer exposures, collection over a broader angular range and/or repeated acquisitions. These various effects of shorter wavelengths converge to improve data accuracy. Experiments using anomalous scattering from elements with absorption edges at ultra-long wavelengths (e.g. S or P) would benefit from the higher accuracy of conventional short or ultra-short wavelength data, whereas the lower accuracy of data collected at ultra-long wavelengths would essentially cancel the interest of stronger anomalous signals. The optimal wavelength for MX experiments is a matter of discussion. We suggest that the range $0.3-0.4 \AA$ is the best choice (albeit shorter wavelengths may be useful for MAD phasing), considering both the increasing ratio of Compton scattering to elastic scattering with photon energy and the current detector technology.

Exploiting the full potential of ultra-short wavelengths would require detectors with a much better DQE than popular MX detectors optimized for conventional wavelengths, such as silicon pixel detectors, or standard CCD detectors with $\mathrm{Gd}_{2} \mathrm{O}_{2} \mathrm{~S}: \mathrm{Tb}$ phosphor. Increasing the thickness of this phosphor coating by a factor of two would increase the DQE by about the same factor, at the expense of a degradation of spatial resolution. The high-pressure beamline ID09A at ESRF (M. Hanfland, private communication) is operating with a Mar555 detector where photons are converted in a fairly thick selenium layer. Such detectors will require further evaluation for MX. Modular CdTe pixel detectors $(\mathrm{Cd}$ and Te $K$-edges at 26.727 and $31.817 \mathrm{keV}$, respectively) are promising. The measured DQE at $35 \mathrm{keV}$ on a prototype $2 \mathrm{~cm} \times 3 \mathrm{~cm}$ imager is $\sim 0.8$ (Medjoubi et al., 2010). Larger detectors are being developed.

Measurements of the degradation of irradiated cryocooled crystals probe essentially effects of primary damage. The behaviour of crystals may be very different at room temperature, and we plan to repeat our experiments on HEWL crystals at room temperature and perform similar measurements on other biomolecular crystals, both cryocooled and at room temperature.

\section{Components of a beamline dedicated to both conventional and high-pressure $M X$ data collection at short wavelength}

MX beamlines can be found at all synchrotron radiation facilities, except those with a low-energy storage ring. The design goals of recent MX beamlines are: (i) wavelength tunability, in order to exploit anomalous phasing, in particular of selenium atoms, albeit that some beamlines are operating at an essentially fixed wavelength; (ii) large CCD or pixel detectors with a good DQE at conventional wavelengths; (iii) automated sample changer; and (iv) remotely controlled operation. So, most beamlines are, not surprisingly, quite similar. Beamlines designed to collect data from microcrystals have some tighter requirements, in particular for overall mechanical stability, goniometer accuracy, focusing optics and sample viewing.

The proposed beamline, based on a different paradigm, is designed for data collection using short- and ultra-shortwavelength X-rays on crystals in either standard conditions (i.e. at ambient pressure) or under high pressure, while preserving access to the whole range of conventional wavelengths. This beamline would extend the possibility of optimized SAD and MAD measurements, encompassing ${ }^{3} K$-edges of all elements with $Z$ from 25 to 58 (i.e. from $\mathrm{Mn}$ to $\mathrm{Ce}$ ). Short and ultra-short wavelengths contribute efficiently to increase the accuracy of measurements, and the beamline itself should be designed in order to contribute to this quest of ultimate accuracy.

Source. With in-vacuum undulators on high-energy storage rings (ESRF, SPring-8, APS, PETRA III), the shortest useful wavelength is below $0.3 \AA$, whereas with intermediate-energy machines, such as DIAMOND and SOLEIL, the practical limit is currently $\sim 0.4 \AA$. Topping-up mode, when available, contributes to high stability. A pair of undulators might be required to cover the whole energy range.

Optics. The main (and perhaps unique) optical element would be a monochromator producing a very stable and parallel beam with a narrow bandpass over the whole energy range. A simple solution could be based on a pair of translatable $\mathrm{Si}(111)$ and $\mathrm{Si}(311)$ channel-cut crystals. Mirrors or other focusing devices would be optional. Optics would be mounted on a heavy table with a vertical translation motion in order to compensate the beam height variation during wavelength change, as implemented on, for example, BM14 at ESRF and PROXIMA 1 at SOLEIL.

Experimental set-up. For conventional MX, the most flexible solution, particularly for measuring accurately anomalous pairs and exploiting the anisotropy of anomalous scattering (Schiltz \& Bricogne, 2008), is a kappa goniometer. In the highpressure mode, the weight and bulk of the DAC are hardly compatible with the kappa goniometer and a second goni-

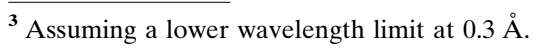


ometer would be required with a single-axis rotation, preferably about a vertical axis, because in this geometry motions of the crystal in the compression cavity of the DAC are minimal during data collection. Using the six-axis robotic arm (normally used as sample changer) to handle the DAC and act as goniometer would be an elegant solution allowing to switch readily from normal operation (with the kappa goniometer) to high-pressure mode (Girard et al., 2010a).

Two items are required for high-pressure experiments: an optical system, coaxial with the X-ray beam, ensuring both the collection of ruby fluorescence signal and sample viewing (e.g. PRL from Betsa, Nangis, France); and a device for programmed pressure ramping or pressure cycling (in view of sample annealing), with monitoring based on the actual pressure in the compression cavity. This system is being tested on the ESRF ID27 beamline.

Detector. The main characteristics of a detector for this beamline would be a good DQE over the whole energy range, a low intrinsic noise, a large area $(>30 \mathrm{~cm} \times 30 \mathrm{~cm})$ and fast readout.

\section{Summary and conclusions}

HPMX is now a method that is technically mature and has been applied to a variety of biomolecular crystals. In particular, high pressure can be used to promote and trap higherenergy conformers of biological relevance, and the feasibility of such studies in the crystalline state is being investigated.

The successful use of ultra-short wavelengths for HPMX led us to investigate the interest of wavelengths shorter than usual in conventional crystallography. Such wavelengths improve not only data quality but also the intrinsic (i.e. assuming an ideal detector) DCE. It is likely that these conclusions will extend to other cryocooled biomolecular crystals and higher photon energies. On the basis of present results, we suggest a new paradigm for synchrotron radiation beamlines. For such beamlines, the crucial importance of detectors with a reasonable efficiency at short wavelengths is underlined.

\section{References}

Akasaka, K. (2006). Chem. Rev. 106, 1814-1835.

Ascone, I., Kahn, R., Girard, E., Prangé, T., Dhaussy, A.-C., Mezouar, M., Ponikwicki, N. \& Fourme, R. (2010a). J. Appl. Cryst. 43, 407416.

Ascone, I., Savino, C., Kahn, R. \& Fourme, R. (2010b). Acta Cryst. D66, 654-663.

Fourme, R., Girard, E., Kahn, R., Ascone, I., Mezouar, M., Dhaussy, A.-C., Lin, T. \& Johnson, J. E. (2003). Acta Cryst. D59, 1767-1772.

Fourme, R., Girard, E., Kahn, R., Dhaussy, A.-C. \& Ascone, I. (2009). Ann. Rev. Biophys. 38, 153-167.

Fourme, R., Kahn, R., Mezouar, M., Girard, E., Hoerentrup, C., Prangé, T. \& Ascone, I. (2001). J. Synchrotron Rad. 8, 1149-1156.

Girard, E., Dhaussy, A.-C., Couzinet, B., Chervin, J.-C., Mezouar, M., Kahn, R., Ascone, I. \& Fourme, R. (2007a). J. Appl. Cryst. 40, 912918.

Girard, E., Fourme, R., Ciurko, R., Joly, J., Bouis, F., Legrand, P., Jacobs, J., Dhaussy, A.-C., Ferrer, J.-L., Mezouar, M. \& Kahn, R. (2010a). J. Appl. Cryst. 43, 762-768.

Girard, E., Kahn, R., Mezouar, M., Dhaussy, A.-C., Lin, T., Johnson, J. E. \& Fourme, R. (2005). Biophys J. 88, 3562-3571.

Girard, E., Marchal, S., Perez, J., Finet, S., Kahn, R., Fourme, R., Marassio, G., Dhaussy, A.-C., Prangé, T., Giffard, M., Dulin, F., Bonneté, F., Lange, R., Abraini, J. H, Mezouar, M. \& Colloc'h, N. (2010b). Biophys. J. 98, 2365-2373.

Girard, E., Prangé, T., Dhaussy, A.-C., Migianu, E., Lecouvey, M., Chervin, J. C., Mezouar, M., Kahn, R. \& Fourme, R. (2007b). Nucl. Acids Res. 35, 4800-4808.

Gonzalez, A., Denny, R. \& Nave, C. (1994). Acta Cryst. D50, 276-282.

Helliwell, J. R., Ealick, S., Doing, P., Irving, T. \& Szebenyi, M. (1993). Acta Cryst. D49, 120-128.

Kabsch, W. (1993). J. Appl. Cryst. 26, 795-800.

Kundrot, C. E. \& Richards, F. M. (1987). J. Mol. Biol. 193, 157-170.

Medjoubi, K., Bucaille, T., Hustache, S., Bérar, J.-F., Boudet, N., Clemens, J.-C., Delpierre, P. \& Dinkespiler, B. (2010). J. Synchrotron Rad. 17, 486-495.

Schiltz, M. \& Bricogne, G. (2008). Acta Cryst. D64, 711-729.

Schiltz, M., Kvick, A., Svensson, O. S., Shepard, W., de La Fortelle, E., Prangé, T., Kahn, R., Bricogne, G. \& Fourme, R. (1997). J. Synchrotron Rad. 4, 287-297.

Shimizu, N., Hirata, K., Hasegawa, K., Ueno, G. \& Yamamoto, M. (2007). J. Synchrotron Rad. 14, 4-10.

Urayama, P., Phillips, G. N. Jr \& Gruner, S. M. (2002). Structure, 10, $51-60$.

Zeng, X., Birrien, J. L., Fouquet, Y., Cherkashov, G., Jebbar, M., Querellou, J., Oger, P., Cambon-Bonavita, M.-A., Xiao, X. \& Prieur, D. (2009). ISME J. 3, 873-876. 